We determined alleles of HLA DQA1 locus by the amplification/restriction procedure described previously [5] with some modifications. This method allowed us to distinguish seven alleles or allele sets (Table 1).

The most "diabetogenic" DQA $1 * 0301$ allele is more frequent in Yakuts (18 from 50 chromosomes) than in Russians (14 from 114 chromosomes). Investigation of Yakut patients with Viliuisk encephalomyelitis uncovered even greater frequency of this allele. Our unpublished investigations together with other data support the idea that presence of DQA $1^{*} 0301$ allele in one of the homologous chromosomes is sufficient for diabetes susceptibility. Thus, comparison of "diabetogenic" allele carrier numbers is probably more informative than comparison of allele frequencies (Table 1 ).

The difference between frequencies of DQA $1 * 0301$ allele carriers in Yakuts and Russians is reliable $(p<0.05$ after correction for multiple comparisons) and the data are in accordance with investigations of HLA DQA1 alleles in Japanese and Chinese, who also have a high frequency of the HLA DQA1*0301 allele and a low incidence of IDDM $[6,7]$. It is possible that role of the DQ $\alpha$ chain is less than is proposed here. However there is no reason to reject the hypothesis of a functional role for DQ loci in diabetes susceptibility. Moreover, there is an association of IDDM with the DQA $1 * 0301$ allele in Japanese subjects. More important are exclusions from the correlation between IDDM incidence and non-Asp57 allele frequency of DQB1 in different populations $[6,8]$. IDDM is a multifactorial disease, and another genetic or environmental factor could play a key role in its resistance in Yakut and other populations. Unusual data concerning the role of $D Q$ genes in diabetes susceptibility are mainly derived from investigations of Mongoloid populations living in a quite different environment. It appears that another race-specific genetic factor is responsible for incidence of IDDM in different populations. In any case further population study of IDDM suscepti-

\section{HLA-A alleles and susceptibility to IDDM}

Dear Sir,

We were interested to read "A gene in the HLA class I region contributes to susceptibility to IDDM in the Finnish population?" [1]. Data from such a large, well-characterized population are extremely valuable in the study of the contribution of HLA genes to insulin-dependent diabetes mellitus (IDDM). We believe, however, that considerable caution should be exercised in the interpretation of the results.

The authors show that of the four Cw1, B56, DR4, DQ8 haplotypes identified, only the A2-associated haplotype was significantly associated with IDDM (82 of 1492 diabetic haplotypes were $\mathrm{A} 2, \mathrm{Cw} 1, \mathrm{~B} 56, \mathrm{DR} 4, \mathrm{DQ} 8$ compared with 14 of 1254 control haplotypes). This finding is taken as evidence that the HLA class I region contributes to diabetes susceptibility. It should be noted, however, that the three non-A2, Cw1, B56, DR4, DQ8 haplotypes are very uncommon, together constituting only 7 of 1492 diabetic haplotypes and 2 of 1254 control haplotypes. This study population, although large, is too small to exclude a disease-predisposing effect of the non- $\mathrm{A} 2$ haplotypes. If the distribution of the A2-positive

Corresponding author: Dr. D. Jenkins, University of Birmingham, Department of Medicine, Queen Elizabeth Hospital, Edgbaston, Birmingham, B15 2TH, UK bility genes is important for better understanding of IDDM aetiology.

Sincerely yours,

I.V.Mersiyanova, V.L.Osakovsky, Yu.A. Knyazev, O.V. Evgrafov

\section{References}

1. Deschamps I, Beressi JP, Khalil I, Robert JJ, Hors J (1991) The role of genetic predisposition to type I (insulin-dependent) diabetes mellitus. Ann Med 23: 427-435

2. Dorman JS et al. (1990) Worldwide differencies in the incidence of type I diabetes are associated with amino acid variation at position 57 of the HLA DQ beta chain. Proc Natl Acad Sci USA 87: 7370-7374

3. Knyazev YuA (1991) Epidemiology of children's diabetes in the USSR. Pediatrics 2: 7-10 (in Russian)

4. Annual Demographic Report of the USSR (1990) Finances and Statistics. Moscow (in Russian)

5. Maeda M, Murayama N, Ishii H et al. (1989) A simple and rapid method for HLA DQA1 genotyping by digestion of PCR-amplified DNA with allele specific restriction endonuclease. Tissue Antigens 34: 290-298

6. Penny NA, Jenkins D, Mijovic CH et al. (1992) Susceptibility to insulin-dependent diabetes mellitus in a Chinese population: role of HLA class II alleles. Diabetes 41: 914-919

7. Todd JA, Mijovic C, Fletcher J et al. (1990) The A3 allele of the HLA DQA1 locus is associated with susceptibility to type I diabetes in the Japanese. Proc Natl Acad Sci USA 87: 1094-1098

8. Awata T, Kuzuya T, Matsuda A et al. (1990) High frequency of aspartic acid at position 57 of HLA-DQ $\beta$ chain in Japanese IDDM patients and nondiabetic subjects. Diabetes 39: $266-269$

Table 1. Distribution of Cw1, B56, DR4, DQ8 haplotypes between diabetic and control subjects

\begin{tabular}{lll}
\hline & $\begin{array}{l}\text { Diabetic } \\
\text { haplotypes } \\
n=89\end{array}$ & $\begin{array}{l}\text { Control } \\
\text { haplotypes } \\
n=16\end{array}$ \\
\hline A2 haplotypes & 82 & 14 \\
Non-A2 haplotypes & 7 & 2 \\
\hline
\end{tabular}

$\chi^{2}=0.016$ (Yates' correction), not significant

and A2-negative Cw1, B56, DR4, DQ8 haplotypes is examined as in Table 1 , one finds that the frequency of $\mathrm{A} 2$ does not differ significantly between the diabetic and control haplotypes. These data, therefore, do not suggest that the HLA class I region makes a particular contribution to susceptibility to IDDM.

The authors also point out that certain Cw3, B62, DR4, DQ8 haplotypes and certain Cw7, B8, DR3, DQ2 haplotypes are significantly associated with IDDM but others are not, depending on the allele at the HLA-A locus. It should be noted, however, that those haplotypes which are not associated with diabetes are uncommon, each non-associated haplotype constituting less than $1 \%$ of both the diabetic and control populations. A similar comparison to that made in Table 1 of the distribution between the diabetic and control subjects of the disease-associated $\mathrm{Cw} 3, \mathrm{~B} 62$, DR4, DQ8 haplotypes (A2, A3 and A24) with those which are not (A1, A11 and A28) gives a 
chi-square value of $5 \times 10^{-4}$, (not significant). Comparison of the distribution of $\mathrm{Cw} 7, \mathrm{~B} 8, \mathrm{DR} 3, \mathrm{DQ} 2$ haplotypes yields a chi-square value of $6 \times 10^{-3}$ (not significant). If HLA-A really does contribute to diabetes susceptibility in addition to the other loci on the extended HLA haplotype, a much larger study would be required to demonstrate statistical significance.

The authors state correctly that a powerful method of analysing whether HLA-A alleles predispose to IDDM independently of class II alleles is to examine the distribution of class I alleles in those diabetic subjects who possess neither of the high-risk class II alleles encoding DR3 and DR4. The authors found that 47 of 55 diabetic subjects possess two copies of the high-risk HLA-A alleles compared with 40 of 58 control subjects, $\chi^{2}=4.33,(p<0.05)$. This weak association is interesting but the authors do not show the data for the remaining subjects who possess one or none high-risk HLA-A alleles. Unless there is any reason to suggest that high-risk HLA-A alleles predispose to IDDM recessively we believe that the entire distribution of high-risk A alleles should be examined before any deductions concerning their effect on diabetes susceptibility in non-DR3/non-DR4 subjects can be made.

Although HLA class I genes are attractive candidate determinants of diabetes susceptibility, we believe that more data are required to test the hypothesis adequately. Given that the population studied by Fennessy et al. [1] is large and wellcharacterized, we suspect that alternative approaches need to be devised to determine whether different closely-related HLA loci make distinct contributions to susceptibility to IDDM in the Finnish population.

Yours sincerely,

D. Jenkins, C. Mijovic

\section{Reference}

1. Fennessy M, Metcalfe K, Hitman GA et al. (1994) A gene in the HLA class I region contributes to susceptibility to IDDM in the Finnish population. Diabetologia 37: 937-944

\section{HLA-A associations with IDDM - a case of numbers?}

\section{Dear Sir,}

The genetic risk for insulin-dependent diabetes mellitus (IDDM) conferred by the HLA class II genes cannot be entirely explained by differences at position 52 of the DQa chain and position 57 of the $\mathrm{DQ} \beta$ chain. Risk conferred by different HLA-DQA1/B1 haplotypes with the same amino acids in these positions varies, as does the risk conferred by the same DQ haplotype occurring in combination with different HLADR alleles [1]. Several explanations have been given, including the influence of other linked genes [1,2], and quantitative differences in HLA class II expression [3]. Fennessy et al. [4] suggest that the risk is influenced by gene(s) telomeric to HLA-C, at or in the vicinity of HLA-A. This region of the MHC has only recently been directly implicated in IDDM susceptibility [5].

This issue is addressed by analysing data from the Finnish cohort of IDDM families, the largest studied for HLA-A, $-\mathrm{C}$, -B, -DR, -DQ haplotypes. They examine individual haplotypes differing only in the HLA-A region and show an association with IDDM only when certain HLA-A alleles are present. They also demonstrate a weak association of these HLA-A alleles with IDDM in non-DR3/non-DR4 patients and conclude that these HLA-A alleles modulate disease susceptibility. From a statistical standpoint, however, their conclusions cannot be supported. Although they show that the CW1 B56 DR4 DQ8 haplotype is significantly associated with IDDM when HLA-A2 is present on this haplotype, other HLA-A alleles are found too infrequently on this haplotype for statistical analysis, and association for these HLA-A alleles cannot be excluded. While the A2 containing haplotype was found on 96 occasions, there are only nine non-A2 CW1 B56 DR4 DQ8 haplotypes in their study, and clearly there is no statisti$\mathrm{cal}$ power to determine association in the non-A2 haplotypes. It cannot, therefore, be concluded that HLA-A2 confers sus-

Corresponding author: Dr. E. Bonifacio, Department of Internal Medicine, Istituto Scientifico San Raffaele, via Olgettina 60, I-20132 Milan, Italy ceptibility, nor that it increases the susceptibility conferred by CW1 B56 DR4 DQ8 alone. Indeed, if a statistical comparison is made between the risk conferred by A2 CW1 B56 DR4 DQ8 and non-A2 Cw1 B56 DR4 DQ8 haplotypes, there is no significant difference for any of the haplotypes studied. The same is also true for the comparisons of HLA-A allele contribution to risk in the two most frequently found diabetic haplotypes in their population, CW3 B62 DR4 DQ8 and CW7 B8 DR3 DQ2. Statistically significant association with IDDM is seen only in haplotypes which are frequently represented, and again there is no difference between the risk conferred by haplotypes with different HLA-A alleles. The weak association of claimed susceptible HLA-A alleles with IDDM in non-DR3/non-DR4 individuals is also dubious since statistical significance is not maintained if a two-tailed chi-square analysis is performed. Moreover, while the authors claim large variation in genetic risk for IDDM for different DQ8 containing haplotypes in Finland $[4,6]$, none of those represented in this [4] and their previous report [6] have statistically different risks for IDDM.

One of the more remarkable findings of the HLA analysis of the Finnish DiMe study is the predominance of relatively few "conserved" HLA-A, -C, -B, -DR, -DQ haplotypes [6]. This conservation of haplotypes, emphasising the stability and low recombination within this stretch of DNA, complicates analyses of contributions to disease risk by separate loci. Fennessy and colleagues have attempted such an analysis, but even in the large DiMe study the high degree of polymorphism at each locus together with haplotype conservation has resulted in too few numbers for statistical comparison. Unfortunately, this is likely to be true for similar analyses at other loci within the MHC. While there are several good reasons why we should consider the importance of HLA class I in the genetic susceptibility and pathogenesis of IDDM, it appears unlikely that classic population-based studies will reach sufficient numbers to clarify a contribution to the genetic risk for IDDM by HLA-A. This region cannot yet be regarded as, nor excluded from, modulating IDDM susceptibility.

Yours sincerely, E. Bonifacio 